\title{
Higher Frequency of Transfusion-Transmitted Virus (TTV) in HIV Patients in Comparison with Healthy Blood Donors
}

\author{
Mohammad Taheri, ${ }^{1}$ Mohammad Motamedifar, ${ }^{1,2,}{ }^{*}$ Jamal Sarvari, ${ }^{1}$ Ramin Yaghobi, ${ }^{3}$ Negin Nikouyan, ${ }^{1}$ \\ Neda Pirbonyeh, ${ }^{1}$ and Kamran Bagheri Lankarani ${ }^{4}$ \\ ${ }^{1}$ Department of Bacteriology and Virology, Shiraz University of Medical Sciences, Shiraz, IR Iran \\ ${ }^{2}$ Shiraz HIV/Aids Research Center, Institute of Health, Shiraz University of Medical Sciences, Shiraz, Iran \\ ${ }^{3}$ Shiraz Transplant Research Center, Shiraz, IR Iran \\ ${ }^{4}$ Health Policy Research Center, Institute of Health, Shiraz University of Medical Sciences, Shiraz, IR Iran \\ "Corresponding author: Mohammad Motamedifar, Department of Bacteriology and Virology, School of Medicine, Shiraz University of Medical Sciences, 71348-45794 Shiraz, IR \\ Iran. Tel/Fax: +98-7132304356, E-mail: motamedm@sums.ac.ir
}

Received 2015 September 12; Revised 2016 April 10; Accepted 2016 November 29.

\begin{abstract}
Background: Epidemiological studies have reported commonly distributed transfusion-transmitted virus (TTV) in different populations with parental risk factors, including human immunodeficiency virus (HIV).

Objectives: This study was performed to determine and compare the prevalence of TTV infection among HIV-positive patients and healthy blood donors.

Patients and Methods: A total of 186 HIV patients and 165 healthy blood donors with no markers of HIV infection were included in this study during 2004 - 2012. Semi-nested Polymerase Chain Reaction (PCR) assay was performed for the detection of TTV DNA. HBV surface antigen (HBsAg), Hepatitis C Virus Antibody (HCVAb), and CD4 were investigated in the sera of HIV-positive subjects. Aspartate transaminase (AST) and Alanine transaminase (ALT) levels were also measured.

Results: The mean age of HIV-positive and healthy subjects was 39.3 and 40.5 years, respectively. In total, 182 (97.9\%) subjects were male and 4 (2.1\%) were female. TTV DNA was detected in 35 out of $186 \mathrm{HIV}$ patients (18.8\%; 95\% CI, $13.2-24.4 \%$ ). The prevalence of TTV in the HIV group was significantly higher $(\mathrm{P}=0.027)$ than blood donors $(11 \%$; $95 \% \mathrm{CI}, 6.2-15.8 \%)$. Age, marital status, unsafe sexual activity, and use of injection drugs were not significantly associated with the prevalence of TTV infection in HIV patients.

Conclusions: Considering the higher frequency of TTV infection in HIV patients in comparison to healthy blood donors, HIV infection may be an important risk factor for TTV infection. In addition, the lower frequency of TTV infection in healthy individuals in comparison to HIV patients reveals the transmission of TTV infection via routes other than blood and drug injection; therefore, the effect of fecal-oral route needs to be examined in future studies.
\end{abstract}

Keywords: TTV, HIV, Blood donors

\section{Background}

Transfusion-transmitted virus (TTV) is a novel unenveloped DNA virus, isolated first from a patient with posttransfusion hepatitis of unknown etiology in Japan $(1,2)$. TTV is a circular, single-stranded DNA virus with a total genomic length of nearly $3.8 \mathrm{~kb}$ (3). It has been classified in the Anelloviridae family, Aphatorquevirus genus (4). TTV genome sequences have been detected in the sera and liver tissues of infected patients, suggesting the involvement of TTV in some acute and chronic liver diseases with unknown etiologies (5).

According to epidemiological studies, TTV is widely distributed among different populations with parenteral risk factors, including hemodialysis (46\%), intravenous drug use (19\% - 40\%), and hemophilia (27.4\% - 68\%). TTV has been also detected at a lower prevalence in voluntary blood donors (1.9\% - 12\%) (6). As recently reported by Prescott and
Simmonds, TTV prevalence ranges from 7\% in Sudan to $83 \%$ in Gambia among rural populations (7).

The prevalence of TTV infection has been also reported to be high among patients with liver diseases, including hepatocellular carcinoma (39\%), non-A to -G liver disease (46\%), non-A to -G fulminant hepatitis (47\%), and cirrhosis (48\%) (8). In addition, the prevalence of TTV DNA in blood donors and thalassemia patients has been estimated at $20 \%$ and $57.2 \%$ in Iran, respectively (9).

The possible association of TTV infection with human pathologies has been debated since the discovery of TTV.Although this infection is possibly associated with some diseases, such as hepatitis, cancer, respiratory diseases, hematological disorders, and HIV infection, there are controversies about the disease-causing potential of TTV in human populations due to its ubiquity. In fact, only certain TTV genotypes and species may be particularly pathogenic un- 
der certain conditions (8). Since the beginning of HIV/AIDS epidemics, approximately 60 million people have been reported to have HIV, and nearly 25 million people have died, thereby indicating the importance of this epidemic $(10,11)$.

There are limited reports on the prevalence of TTV in patients with HIV infection $(12,13)$. In this regard, a prevalence study including a small number of HIV patients suggested a slightly increased prevalence, compared to blood donors. Puig-Basagoiti et al. described a high TTV prevalence in a large HIV population in Spain (14). Moreover, a Danish cohort study reported a prevalence of $76 \%$ among HIV patients and suggested TTV as an independent factor for patient survival (13).

Based on previous studies, there is an important relationship between HIV-TTV coinfection and suppression of the immune system. Moreover, a higher TTV titer was reported in HIV patients with lower CD4 counts (15). Nevertheless, the prevalence of TTV infection in HIV patients has not been yet reported in Shiraz, Iran.

\section{Objectives}

This cross sectional study was carried out to determine the prevalence of TTV infection in HIV patients, referring to an HIV referral center in Shiraz, Iran, and to compare it with healthy blood donors.

\section{Patients and Methods}

In total, 186 patients were recruited among all clients admitted to Shiraz voluntary counseling and testing (VCT) center, affiliated to Shiraz University of Medical Sciences (Shiraz, Iran) during 2004 - 2012. All the subjects were recruited voluntarily, and informed consents were obtained. The required data were collected from the medical records of patients with confirmed HIV infection at Shiraz VCT center. Serum samples were also obtained from HIV patients. Moreover, 165 healthy blood donors who had no markers of HIV infection were included in the study as the control group. This study was approved by the vice-chancellor for research (No.5565), Shiraz University of Medical Sciences.

All the serum samples were stored at $-70^{\circ} \mathrm{C}$ before TTV DNA detection. Viral DNA was extracted from the serum, using RTP DNA/RNA Virus Mini Kit (Invitek, Freiburg, Germany), according to the manufacturer's instructions. The purified DNA was then eluted from the membrane with 50 $\mu \mathrm{L}$ of elution buffer and stored at $-20^{\circ} \mathrm{C}$ until performing a semi-nested polymerase chain reaction (PCR) assay, as described by Okamoto et al. (14).

The sequences of primers used in this study are presented in Table 1. The first round of semi-nested PCR was performed in a total volume of $25 \mu \mathrm{L}$, containing $1 \mathrm{X}$ reaction buffer (CinnaGen, Iran), $200 \mu \mathrm{M}$ of each dNTP (CinnaGen, Iran), 1U Taq DNA polymerase (CinnaGen, Iran), $0.5 \mu \mathrm{M}$ of R1 and $\mathrm{F} 1$ specific primers, $1.5 \mathrm{mM}$ of $\mathrm{MgCl}_{2}$ (CinnaGen, Iran), and $5 \mu \mathrm{L}$ of extracted DNA. In the second round, 5 $\mu \mathrm{L}$ of the first-round PCR product was added to the master mix, which contained R1 and F2 specific primers.

Nested PCR was performed as follows: initial denaturation at $94^{\circ} \mathrm{C}$ for 10 minutes, 30 cycles of denaturation at $94^{\circ} \mathrm{C}$ for 45 seconds, annealing at $60^{\circ} \mathrm{C}$ for 45 seconds, extension at $72^{\circ} \mathrm{C}$ for 45 seconds, and final extension at $72^{\circ} \mathrm{C}$ for 5 minutes. The annealing temperature of the second round was $59^{\circ} \mathrm{C}$. The amplified products were run on $2 \%$ agarose gel in a buffer, containing $0.5 \mu \mathrm{g} / \mathrm{mL}$ of ethidium bromide for visualizing a 271-bp TTV-specific band under a UV light transilluminator.

Aspartate transaminase (AST) and alanine transaminase (ALT) measurements were also performed for all HIV patients and healthy control blood donors. In addition, CD4 cell count was measured via flow cytometry (Partec, Germany). HBV surface antigen (HBsAg; Siemens Healthcare Diagnostics, Tokyo, Japan) and hepatitis C virus antibody (HCVAb; Diapro, Milan, Italy) were detected by commercial ELISA kits, according to the manufacturer's instructions.

Intergroup comparisons were made, using Chi square or Fisher's exact test for categorical variables and student's t test or Mann-Whitney and Kruskal-Wallis tests (when appropriate) for quantitative variables, using SPSS version 15 (SPSS Inc., Chicago, IL, USA). To determine the independent effect of different viruses on changes in ALT level, analysis was adjusted in a logistic regression model. P value less than 0.05 was considered statistically significant.

\section{Results}

In total, 186 (male, 182; female, 4) HIV patients and 165 (male, 160; female, 5) healthy individuals were enrolled in this study. The mean age of HIV patients and healthy subjects was 39.3 and 40.5 years, respectively. The mean age of TTV-positive and -negative subjects in the HIV group was 39.4 and 39.3 years, which was not significantly different $(P$ $=0.69$ ). In the control group, the mean age of TTV-positive and -negative subjects was 36.7 and 40.9 years, which was not significantly different $(\mathrm{P}=0.12)$. The male-to-female ratio was 182:4 in the HIV group and 160:5 in the healthy control group. However, the limited number of female subjects did not allow us to analyze the association between gender and TTV infection in the groups. The prevalence of TTV infection in HIV-positive subjects in relation to the presumed source of infection is summarized in Table 2. 
Table 1. Primers Used to Amplify ORF1(VP1) Gene of TTV

\begin{tabular}{|c|c|c|}
\hline & Primer & Product Size, bp \\
\hline \multirow{2}{*}{ First round } & (R1) $5^{\prime}$-CTG GCA TTT TAC CAT TTC CAA AGT T-3 ${ }^{\prime}$ & \multirow{2}{*}{286} \\
\hline & (F1) $5^{\circ}$-ACA GAC AGA GGA GAA GGC AAC ATG-3' & \\
\hline \multirow{2}{*}{ Second round } & (R2) $5^{-}$-CTG GCA TTT TAC CAT TTC CAA AGT T-3 ${ }^{-}$ & \multirow{2}{*}{271} \\
\hline & (F2) ('-GGC AAC ATG TTA TGG ATA GAC TGG-3’' & \\
\hline
\end{tabular}

Table 2. The Main Characteristics of HIV Patients According to TTV Infection

\begin{tabular}{|c|c|c|c|c|}
\hline Variables & HIV Patients & TTV Positive & TTV Negative & P Value \\
\hline No. & 186 & $35(18.8)$ & $151(81.2)$ & 0.027 \\
\hline Mean age, $y$ & 39.36 & 39.40 & 39.35 & 0.12 \\
\hline Marital status & 109 & $22(21.8)$ & $87(79.2)$ & 0.35 \\
\hline Unsafe sexual activity & 116 & $24(20.6)$ & $92(79.4)$ & 0.26 \\
\hline Intravenous drug use & 123 & $23(18.7)$ & $100(81.3)$ & 0.55 \\
\hline History of blood transfusion & 14 & $4(28.6)$ & $10(71.4)$ & 0.28 \\
\hline History of imprisonment & 89 & $14(15.5)$ & $75(74.5)$ & 0.20 \\
\hline HIV/HBV coinfection & 120 & $24(20)$ & $96(20)$ & 0.36 \\
\hline HIV/HCV coinfection & 19 & $6(31.5)$ & $13(68.5)$ & 0.19 \\
\hline Mean ALT level (IU/L) & 9.15 & 8.08 & 9.39 & 0.24 \\
\hline Mean AST level, IU/L & 13.64 & 14.37 & 13.47 & 0.72 \\
\hline Mean CD4 count, cell $/ \mu \mathrm{L}$ & $3.3444 \mathrm{E} 2$ & $3.3020 \mathrm{E} 2$ & $3.3542 \mathrm{E} 2$ & 0.73 \\
\hline
\end{tabular}

TTV DNA was detected in 35 out of 186 (18.8\%; 95\% CI, 13.2 - 24.4\%) HIV patients, which was significantly higher ( $\mathrm{P}$ $=0.027$ ) than blood donors (18 out of 165, 11\%; 95\% CI, 6.2 $15.8 \%$ ). Age, marital status, unsafe sexual activity, and injection drug use were not associated with the prevalence of TTV infection in the HIV group.

According to the findings, ALT and AST levels were not significantly different among subjects with and without TTV in either of the groups. Overall, the mean levels of ALT and AST in HIV-positive and -negative groups showed a significant difference $(\mathrm{P}<0.001)$. Statistical analysis of the mean CD4 cell count in TTV-positive and -negative HIV patients did not show a significant difference between the groups. In addition, the effects of HBV and HCV infections on TTV were not significant in HIV patients.

\section{Discussion}

Recent studies have suggested that TTV infection is a relatively common viral infection among different highrisk groups around the world, including those with bloodborne viruses (16) such as HIV(13). Based on several studies, there is a significant association between HIV-TTV coinfection and immune system suppression (15). Based on our findings, the frequency of TTV infection was significantly higher in HIV patients in comparison with the healthy controls.

In a cross sectional study by Sherman et al. in the United States, $38 \%$ of HIV patients and $14.4 \%$ of HIVnegative controls were positive for TTV infection (17). Additionally, Puig-Basagoiti et al. reported a high prevalence of TTV infection in a large HIV-infected group in Spain (12). Moreover, in a cohort study by a Danish researcher, TTV DNA was detected in $76 \%$ of HIV patients, and presence of TTV was introduced as an independent factor for patient survival (13).

According to previous reports, TTV changes are independent of CD4 count in HIV infection. Patients with TTV tend to show lower HIV viral loads. On the other hand, Christensen et al. proposed that low CD4 count is associated with a high TTV titer, which is an independent predictor of survival (13). The clinical significance of TTV in HIV infection remains unknown. In this regard, Garcia et al. did not find a significant correlation between the increased frequency of TTV infection (or viral load) and CD4+ 
cell count or HIV stage (18).

HIV infection may be involved in TTV immune evasion due to HIV-related immune dysfunction, resulting in higher replication rates and persistence (19). It has been also reported that management of HIV replication and CD4+ count is associated with liver disease progression $(20,21)$. Moreover, a higher frequency of TTV DNA in the HIV group suggested that this population is at a higher risk of infection; it may also play an important role in TTV transmission.

The frequency of TTV infection is higher in high-risk groups with HBV, HCV, hemophilia, hemodialysis, or intravenous drug use, compared to healthy individuals; this finding might be associated with the route of TTV transmission (22). According to many studies, coinfection of TTV with either HBV or HCV is common, while the significance of TTV infection in patients with chronic HBV or HCV infection is unclear $(21,22)$. The results of the present study showed no significant difference in the rate of TTV infection among HIV patients and those with HIV/HBV or HIV/HCV coinfection.

Some evidence suggests that TTV infection is associated with higher levels of ALT and AST in HCV/HIVcoinfected patients, compared to those with HIV or HCV alone (23). In this study, the levels of ALT and AST in the HIV/HCV and HIV/HBV groups were not significantly different from patients with only HIV infection. However, ALT and AST levels were significantly higher among HIV patients, compared to the healthy group. This finding might be either associated with the higher frequency of TTV in the HIV group or result from HBV and HCV coinfection in this group. On the other hand, TTV coinfection with HCV was associated with chronic liver disease $(8,24)$, suggesting that TTV could increase the risk of HCV-related liver cirrhosis and cause further complications. However, other reports have not demonstrated the effects of coinfection with TTV on the clinicopathological course of chronic HCV (25).

Overall, the prevalence of TTV infection among healthy subjects varies in different areas (8). Molecular epidemiological studies have shown a prevalence of $36 \%$ in Thailand, $12 \%-19 \%$ in Japan, $1 \%$ in the United States, $2 \%-10 \%$ in European countries, and $7.4 \%$ in India (8). Moreover, previous studies from Iran have reported a prevalence of $20 \%$ among blood donors positive for TTV DNA. The results of the present study revealed that $11 \%$ (18 out of 165 ) of healthy subjects were positive for TTV DNA.

The detection rate of TTV-positive sera in the general population varies greatly in different areas, ranging from $5 \%$ in Brazil to more than $90 \%$ in Japan, Pakistan, and Russia $(8,26)$. The high prevalence of TTV infection in healthy individuals may be associated with the mechanisms of TTV immune evasion, which seeks to establish a persistent in- fection in immunocompetent individuals (27).

It has been suggested that TTVs are commensal under normal conditions and do not cause any diseases instantly. However, TTV probably behaves as an opportunistic pathogen and causes damage only under specific circumstances (8). Although it remains unclear how the immune system is involved in the natural course of TTV infection, previous studies have revealed an association between TTV and severe immunodeficiency in HIV patients and other immunocompromised individuals $(13,15)$.

Variations in the frequency of TTV infection among different countries could be a result of heterogeneity and variability in TTV isolates, as well as differences in primer sequences and sensitivity of PCR method. The primers used in this study were similar to those used in the mentioned research, suggesting that variations in TTV prevalence among different countries are not related to differences in the used primers.

TTV is speculated to be transmitted via parenteral, sexual, fecal-oral, breathing, and salivary routes (8). Investigation of risk factors for TTV infection in HIV patients can be important for introducing proper control measures. In the present study, several risk factors, including history of blood transfusion, history of imprisonment, intravenous drug use, unsafe sex, and marital status were surveyed as parameters increasing the risk of TTV infection in HIVpositive patients.

Although TTV infection is more common in polytransfused patients, the present results did not show history of transfusion as a risk factor for TTV infection in HIV patients. Moreover, Sherman et al. reported a TTV prevalence of 50\% among patients with a history of intravenous drug use (17). However, statistical analysis in our study showed that intravenous drug use was not a risk factor for TTV infection in HIV patients.

Considering the presence of TTV in the cervical epithelium and semen, sexual route has been introduced as another possible route of TTV transmission in the human adult population (28). In the present study, marital status and unsafe sexual activity were not significantly associated with the high rate of TTV infection in HIV patients. Although in some studies (8), age was reported as a risk factor for TTV infection, we did not find any association between age and TTV infection in either of the groups.

One limitation of the present study, besides the relatively small sample size, is the lower number of female subjects. Therefore, we could not perform any gender analyses on the frequency of TTV infection in the groups.

In conclusion, the present results demonstrated that TTV infection rates are higher in HIV patients in comparison to healthy blood donors. Therefore, HIV infection may be an important risk factor for TTV infection. Moreover, de- 
tection of TTV infection in $11 \%$ of healthy individuals suggests transmission through routes other than blood and injection (eg, fecal-oral route), causing food and water contamination and infecting the milk of TTV-positive women.

\section{Acknowledgments}

We would like to thank all the participants for their cooperation in this study. This work was financially supported by health policy research center and Shiraz University of Medical sciences, Shiraz, Iran.

\section{Footnotes}

Authors` Contribution: Concept and design: Mohammad Motamedifar and Jamal Sarvari; Acquisition of data: Negin Nikouyan and Neda Pirbonyeh; Statistical analysis and interpretation of data: Mohammad Motamedifar, Ramin Yaghoobi and Mohammad Taheri; Drafting of the manuscript: Mohammad Taheri; Critical revision of the manuscript for important intellectual content: Mohammad Motamedifar and Jamal Sarvari; Study supervision: Mohammad Motamedifar and Kamran Bagheri Lankarani. Funding/Support: This study supported by Department of Bacteriology and Virology, School of Medicine, Shiraz University of Medical Sciences, Shiraz, IR Iran

\section{References}

1. Nishizawa T, Okamoto H, Konishi K, Yoshizawa H, Miyakawa Y, Mayumi M. A novel DNA virus (TTV) associated with elevated transaminase levels in posttransfusion hepatitis of unknown etiology. Biochem Biophys Res Commun. 1997;241(1):92-7. doi: 10.1006/bbrc.1997.7765. [PubMed: 9405239].

2. Vasilyev EV, Trofimov DY, Tonevitsky AG, Ilinsky VV, Korostin DO, Rebrikov DV. Torque Teno Virus (TTV) distribution in healthy Russian population. Virol J. 2009;6:134. doi: 10.1186/1743-422X-6-134. [PubMed: 19735552].

3. Okamoto H, Nishizawa T, Takahashi M, Tawara A, Peng Y, Kishimoto J, et al. Genomic and evolutionary characterization of TT virus (TTV) in tupaias and comparison with species-specific TTVs in humans and non-human primates. J Gen Virol. 2001;82(Pt 9):2041-50. doi: 10.1099/0022-1317-82-9-2041. [PubMed: 11514713].

4. Hussain T, Manzoor S, Waheed Y, Tariq H, Hanif K. Phylogenetic analysis of Torque Teno Virus genome from Pakistani isolate and incidence of co-infection among HBV/HCV infected patients. Virol J. 2012;9:320. doi: 10.1186/1743-422X-9-320. [PubMed: 23270330].

5. Inami T, Konomi N, Arakawa Y, Abe K. High prevalence of TT virus DNA in human saliva and semen. J Clin Microbiol. 2000;38(6):2407-8. [PubMed: 10835017].

6. Gallian P, Berland Y, Olmer M, Raccah D, de Micco P, Biagini P, et al. TT virus infection in French hemodialysis patients: study of prevalence and risk factors. J Clin Microbiol. 1999;37(8):2538-42. [PubMed: 10405397].

7. Prescott LE, Simmonds P. Global distribution of transfusiontransmitted virus. $N$ Engl J Med. 1998;339(11):776-7. doi: 10.1056/NEJM199809103391118. [PubMed: 9742036].
8. Spandole S, Cimponeriu D, Berca LM, Mihaescu G. Human anelloviruses: an update of molecular, epidemiological and clinical aspects. Arch Virol. 2015;160(4):893-908. doi: 10.1007/s00705-0152363-9. [PubMed: 25680568].

9. Sara A, Solhjoo K, Jahromi ARS, Yaghobi R. Study the prevalence of TT virus infection in South Iranian volunteer blood donors. AfrJMicrobiol Res. 2012;6(32):6230-4.

10. Perkins EL, Stennis KB, Taylor Spriggs V, Kwegyir-Afful EA, Prather A. Is Knowledge Enough? Considering HIV/AIDS Risk Behaviors and HIV/AIDS Knowledge with African American Women. Int J High Risk Behav Addict. 2014;3(3):e15038. doi: 10.5812/ijhrba.15038. [PubMed: 25593891].

11. Zareban I, Araban M. Human Immunodeficiency Virus/Acquired Immunodeficiency Syndrome Risk (HIV/AIDS)-Taking Behavior and HIV/AIDS Knowledge With African-American Women. Int J High Risk Behav Addict. 2015;4(4):e23926. doi: 10.5812/ijhrba.23926. [PubMed: 26870711].

12. MacDonald DM, Scott GR, Clutterbuck D, Simmonds P. Infrequent detection of TT virus infection in intravenous drug users, prostitutes, and homosexual men. J Infect Dis. 1999;179(3):686-9. doi: 10.1086/314642. [PubMed: 9952377].

13. Christensen JK, Eugen-Olsen J, S. Lrensen M, Ullum H, Gjedde SB, Pedersen BK, et al. Prevalence and prognostic significance of infection with TT virus in patients infected with human immunodeficiency virus. J Infect Dis. 2000;181(5):1796-9. doi: 10.1086/315440. [PubMed: 10823787].

14. Puig-Basagoiti F, Cabana M, Guilera M, Gimenez-Barcons M, Sirera G, Tural C, et al. Prevalence and route of transmission of infection with a novel DNA virus (TTV), hepatitis C virus, and hepatitis $G$ virus in patients infected with HIV.J Acquir Immune Defic Syndr. 2000;23(1):89-94. [PubMed: 10708061].

15. Shibayama T, Masuda G, Ajisawa A, Takahashi M, Nishizawa T, Tsuda F, et al. Inverse relationship between the titre of TT virus DNA and the CD4 cell count in patients infected with HIV. AIDS. 2001;15(5):563-70. [PubMed: 11316992]

16. Motamedifar M, Taheri M, Lankarani K, Gholami M. The prevalence and risk factors of hepatitis delta virus in HIV/HBV co-infected patients in Shiraz, Iran, 2012. Iran J Med Sci. 2015;40(5):448.

17. Sherman KE, Rouster SD, Feinberg J. Prevalence and genotypic variability of TTV in HIV-infected patients. Dig Dis Sci. 2001;46(11):2401-7. [PubMed: 11713943].

18. Garcia-Alvarez M, Berenguer J, Alvarez E, Guzman-Fulgencio M, Cosin J, Miralles P, et al. Association of torque teno virus (TTV) and torque teno mini virus (TTMV) with liver disease among patients coinfected with human immunodeficiency virus and hepatitis C virus. Eur J Clin Microbiol Infect Dis. 2013;32(2):289-97. doi: 10.1007/s10096-012-1744-1. [PubMed: 22983402].

19. Touinssi M, Gallian P, Biagini P, Attoui H, Vialettes B, Berland Y, et al. TT virus infection: prevalence of elevated viraemia and arguments for the immune control of viral load. J Clin Virol. 2001;21(2):135-41. [PubMed: 11378494].

20. Reiberger T, Ferlitsch A, Sieghart W, Kreil A, Breitenecker F, Rieger A, et al. HIV-HCV co-infected patients with low CD4+ cell nadirs are at risk for faster fibrosis progression and portal hypertension. J Viral Hepat. 2010;17(6):400-9. doi: 10.1111/j.1365-2893.2009.01197.x. [PubMed: 19780945].

21. Thorpe J, Saeed S, Moodie EE, Klein MB, Canadian Co-infection Cohort $S$. Antiretroviral treatment interruption leads to progression of liver fibrosis in HIV-hepatitis C virus co-infection. AIDS. 2011;25(7):967-75. doi: 10.1097/QAD.0b013e3283455e4b. [PubMed: 21330904].

22. Okamoto H, Takahashi M, Nishizawa T, Tawara A, Fukai K, Muramatsu U, et al. Genomic characterization of TT viruses (TTVs) in pigs, cats and dogs and their relatedness with species-specific TTVs in primates and tupaias. J Gen Virol. 2002;83(Pt 6):1291-7. [PubMed: 12029143]. 
23. Shieh B, Chang MJ, Ko WC, Chen EJ, Wu JC, Lee CF, et al. Effects of multiple virus coinfections on disease progression in HIV-positive patients. Intervirology. 2003;46(2):105-13. [PubMed:12684549].

24. Ikeda H, Takasu M, Inoue K, Okamoto H, Miyakawa Y, Mayumi M. Infection with an unenveloped DNA virus (TTV) in patients with acute or chronic liver disease of unknown etiology and in those positive for hepatitis C virus RNA. J Hepatol. 1999;30(2):205-12. [PubMed: 10068097].

25. Masia G, Ingianni A, Demelia L, Faa G, Manconi PE, Pilleri G, et al. TT virus infection in Italy: prevalence and genotypes in healthy subjects, viral liver diseases and asymptomatic infections by parenterally transmitted viruses. J Viral Hepat. 2001;8(5):384-90. [PubMed:
11555197]

26. Pirouzi A, Bahmani M, Feizabadi MM, Afkari R. Molecular characterization of Torque teno virus and SEN virus co-infection with HIV in patients from Southern Iran. Rev Soc Bras Med Trop. 2014;47(3):275-9. [PubMed: 25075476]

27. Maggi F, Bendinelli M. Immunobiology of the Torque teno viruses and other anelloviruses. Curr Top Microbiol Immunol. 2009;331:65-90. [PubMed: 19230558].

28. Zheng MY, Lin Y, Li DJ, Ruan HB, Chen Y, Wu TT. [TTV and HPV coinfection in cervical smears of patients with cervical lesions in littoral of Zhejiang province]. Zhonghua Shi Yan He Lin Chuang Bing Du Xue Za Zhi. 2010;24(2):110-2. [PubMed: 21110428]. 